\title{
L'Élevage dans la Boucle du Niger
}

\author{
par G. DOUTRESSOULLE et TRAORE Saillou, Vétérinaire africain
}

LE fleuve Niger joue un rôle très important dans l'élevage soudanais; il conditionne par ses pâturages de décrue et l'abreuvement du bétail la vie des trois quarts du cheptel.

Après avoir pris sa source dans les contreforts du Fouta-Djallon, en Guinée française, il se dirige vers le nord-est, puis s'incurve après le lac Debo pour former la boucle du Niger.

Nous étudierons l'élevage de cette région, limitée au nord et à l'est jusqu'à Ansongo par le fleuve, à l'ouest par les grands lacs, au sud par les monts de Douentza-Hombori-Anniganda.

\section{LE MILIEU}

Celte région comprise entre $17^{\circ}$ et $16^{\circ} 30$ de latitude nord couvre une superficie d'environ 50.000 kilomètres carrés. Elle appartient à la zone sahelienne caractérisée par la rareté des pluies annuelles (de 200 à $300 \mathrm{~mm}$ ) avec une durée de trois mois (juillet-août-septembre). Elle constitue une des plus belles régions d'élevage de l'A.O.F.
De Tombouctou à Ansongo, le fleuve coule dans un véritable désert que seule sa crue féconde sur une largeur de 4 à 15 kilomètres selon les régions. Il est bordé, jusque vers Tosaye, par des dunes vives à l'aspect désolé. A cet endroit, les rives deviennent rocheuses et se relèvent en falaises à pic pour former un seuil de 300 mètres de largeur. Ce défilé franchi, les dunes réapparaissent jusqu'aux rapides de Fafa, en aval d'Ansongo. Le lit du fleuve est semé d'îles nombreuses, couvertes d'herbe, où se reunissent les troupeaux pendant la saison sèche.

Le régime des eaux dans cette région est simple : à partir de Tombouctou, la crue se déroule régulièrement jusqu'aux rapides de Fafa. Elle débute vers le milieu de juillet, pour atteindre son maximum vers la fin de décembre. Elle décroît rapidement à partir de mars et les eaux sont basses à la fin de juin.

Les deux rives sont dissemblables : la rive gauche a reçu le nom d'Haoussa; quant à la rive droite ou Gourma, elle se continue vers le; sud, dans la

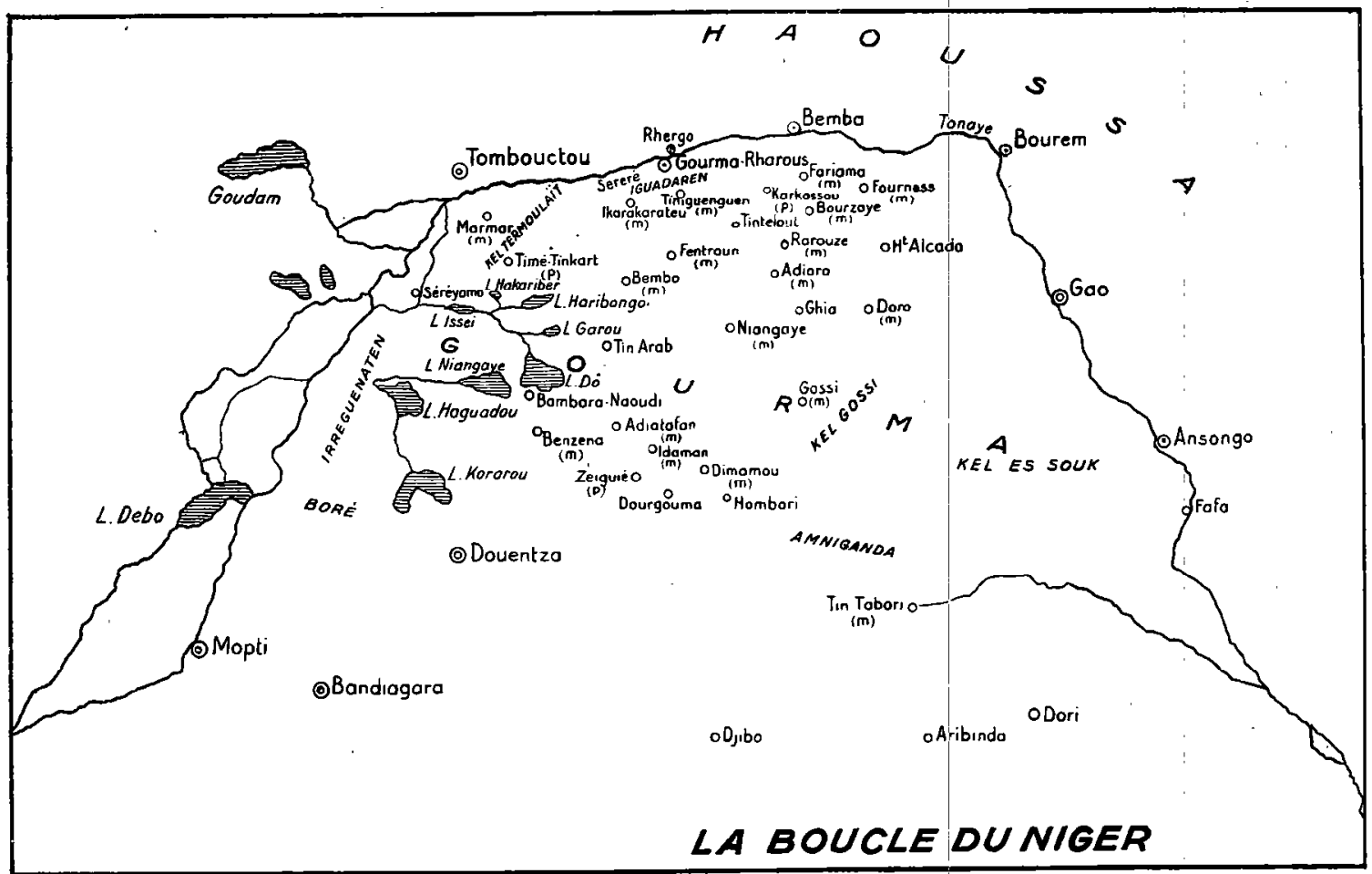


concavité de la vaste boucle que décrit le fleuve, par le plateau central nigérien.

Dans 'la boucle du Niger, à l'est du système des lacs et des régions du Boré et du Korarou, s'étend un vaste plateau formé de surface latéritique entrecoupée de sable et de petits massifs montagneux peu élevés. Il est bordé au sud d'Hombori par la falaise de Bandiagara, continuée à l'est par les hauteurs de l'Anniganda.

Ce plateau, couvrant plus de 15.000 kilomètres carrés. est sans écoulement, sauf au sud-est de la mare de Gossi. Il en résulle que, en hivernage, l'eau s'accumule dans toutes les dépressions, formant des mares, la plupart temporaires, dans le lit desquelles des puits sont creusés en saison sèche. La durée de retenue des eaux varie selon leur importance et leur profondeur, de quelques semaines à six ou huit mois.

La plus importante, la mare de Gossi, située à 90 kilomètres au nord-est d'Hombori, est permanente et couvre, en hivernage, environ 9.000 hectares; la plus grande partie en est couverte, en saisón sèche, d'arbres et de végétation, notamment. de bourgou. Au nord, les mares d'Hékia, Tin Arab et Adiora forment un chapelet, se réunissant après les pluies sur 15 kilomètres de long; dans le lit de ces mares, desséchées dès le mois de janvier, des puits peu profonds permettent de trouver l'eau.

Le centre du plateau est parsemé de mares moins importantes dont les principales sont :

- la mare de Niangaye, qui conserve l'eau jusqu'en avrll ou mai,

- la mare de Fentourou, à 25 kilomètres au' nord, dont le lit, en saison seche, est foré de nombreux puits,

- celle de Ghia, à 40 kilomètres à l'est, qui ne s'assèche qu'en mars,

et l'ouest du plateau avec les mares de Benzéna, Idaman, In Adiatafan qui s'assèchent en mars ou avril.

Il existe à l'ouest du Gourma un système des lacs intérieurs aujourd'hui desséchés (Issei, Courou, Haribongo, Dô, Akhariben, etc.) qu'alimentaient autrefois les eaux du Niger. Depuis quelques années, le barrage de ce fleuve à Sansanding, la déviation d'unepartie de ses eaux pour l'irrigation du Sahel ont complètement désorganisé cet admirable réseau. lagunaire qui faisait de cette partie du Courma une oașis perdue dans un erg et où Bellahs, Ikoulouten, Kountas cultivaient du mil, du blé et même du riz. Les ancieńs lacs sont aujourd'hui transformés en forêt d'épineux, et il y reste assez d'humus pour faire pousser de nombreuses graminées. Les Kountas. les Ballahs Ikoulouten de la tribu des Irreguenaten, quelques fractions de Foulanes Kriabés s'y sont sédentarisés grâce à la permanenco do l'cau dans les puits peu profonds creusés au fond de ces cuvettes naturelles. Les animaux de ces tribus pivotent autour de ce système lacustre pendant toute l'année.

A l'est, faisant suite à la ligne des monts qui, en forme d'arc de cercle se terminent au seuil de Tosaye, le plateau s'incline vers le fleuve et donne naissance en hivernage à de nombreux marigots qui reproduisent sur une petité ćchello los dallols de la rive gauche.

Tout le long du fleuve, le plateau, sur une profondeur de 20 à 30 kilomètres, est bordé par plusieurs lignes de dunes vives. Cette ligne est interrompue, dans la région de Tosaye, par une ligne rocheuse qui se continue sur la rive gauche du Niger et forme un défilé dans lequel passe le fleuve.

\section{L'ÉLEVEUR}

Dans la boucle du Niger et sur le plateau se rencontrent les races Targui, Maure, Peul iet Songhaï.

Les Touareg et'les Maures sont des nomades, les Peuls sont mi-nomades, mi-sédentaires, et les Songhai des sédentaires.

a) Les nomades. - Les Maures sont plus nombreux et représentés par les Kountas, éleveurs et commerçants de bétail, dans la région des lacs Garou et Titalaouen. Les Touareg constituent'le gros des nomades. Parmi eux, nous rencontrons : les Irregueneten, importante tribu avec de nombreuses fractions qui vont de la région de Sareyamou jusqu'au sud de l'Iloa, près de Tombouctou.

Les Kel Temoulait, Imochars du groupe Tad mekett, de la rive droite du fleuve en face Iloa jusqu'au Sereré.

Les Imededren de l'île de Sereré.

Les Igouadaren I et II, Imochars, cousins des Oulliminden du Haoussa qui vivent aux basses eaux, les uns à l'ouest, les autres à l'est de Rharous.

En suivant la rive droite du Niger jusqu'en face de Bourèm, on rencontre en saison sèche quelques petites fractions indépendantes, telles les Chiokhanes de l'est, plusieurs fractions Cheriffen, une partie des Kel-Rheris dépendant de, Gao, les Kel Tagarangot, et de Bourèm à Ansongo, les Idnanes, les Tenguereguedech, les Cheriffen. Dans la boucle entre Hombori, Dori et le fleuve, évoluant surtou autour des quelques mares qui enrichissent cette région, les Kel-Gossi, les Logomoten, les Kel Rheris, les Kel es Souk.

En suivant la falaise de Hombori vers l'ouest existe un important peuplement peul, qui continue en remontant vers le nord et en contournant le plateau à l'ouest jusqu'à la limite des lacs et à Saréyamou. Ce sont les Foulankriabés.

b) Les sédentaires. - Les Songhaïs sont fixés le long du fleuve, sur les berges et dans los îlos, 
groupés par village dont la densité augmente à mesure qu'on descend vers le sud. Ils vivent un peu de pêche, surtout d'agriculture et d'élevage. La vallée du Niger, avec ses crues périodiques, ses nombreuses îles et ses vastes zones d'inondation, offre de grosses ressources pour l'élevage.

L'histoire nous apprend que les Songhaïs firent de cette zone une des plus riches contrées du Soudan. Sa déchéance actuelle date de trois siècles; commencée au dix-septième siècle par l'invasion marocaine, elle s'est continuée jusqu'à notre époque par la dure oppression targuie. Trois siècles d'esclavage ont réduit et abêti ces malheureux Songhaïs, au point que le terme "Koïraboro " (hommes des villages) sous lequel on les désigne, est devenu un terme de mépris.

$\mathrm{Au}$ sud de la région des mares, à Hombori, vit un groupement Songhaï, émigré de Gao avant et après la conquête marocaine et réfugié sur les hauteurs. Il possede des troupeaux et en confie la garde aux bergers peuls. Ce ne sont pas des éle-veurs, mais ils s'intéressent beaucoup à l'élevage du cheval. Chaque famille entretient quelques juments ainsi qu'un cheval qu'ils nourrissent de mil ou de paguiri.

\section{MODE DE VIE}

Le caractère désertique du plateau du Gourma interdit actuellement toute sédentarisation; aussi n'est-il parcouru par les nomades qúe pendant la saison des pluies pour être abandonné ensuite, au fur et à mesure de l'assechement des mares et des puits.

La nomadisation des Touareg est à court rayon. Elle se fait suivant une loi fixe. A la saison des pluies (juillet-novembre), les tribus sont dispersées, car elles trouvent partout eau et pâturages. A mesure que l'eau se raréfie, les tribus se groupent et evoluent de mare en mare, en réservant toujours pour la fin de la saison sèche les points où l'eau reste en dernier lieu : fleuve ou mares permanentes.

Sauf raisons politiques, on trouvera toujours, à un moment donné de l'année, telle tribu à tel point d'eau déterminé.

La composition du cheptel de chaque tribu est en relation avec l'étendue plus ou moins grande de son cercle de nomadisation. Les tribus à petit parcours ont surtout des bceufs, dont le rapport est plus grand; les tribus à grande nomadisation ont davantage de chameaux et de chèvres; les chevaux n'existent qu'en petite quantité

\section{PATURAGES - TRANSEUMANCES ABREUVEMENT}

L'état des pâturages varie beaucoup suivant les diverses périodes de l'année et la végétation hor- bacée et arboricole n'est pas uniforme pour l'ensemble du cercle.

La flore de cette région possède, en raison des conditions climatiques, un caractère tropophile très accusé. Les espèces résistent à la sécheresse prolongée par le développement des parties ligneuses et épineuses et la caducité des feuilles.

La végétation arbustive et herbacée est différente suivant la nature du sol qui se présentc sous trois aspects :

a) Dunes sablonneuses peu élevées, partout fixées par des graminées clairsemées.

b) Plaines argileuses dénuées de végétation, recouvertes d'eau en hivernage et desséchées; fendillées en saison seche.

c) Plateaux de latérite à production arbustivé,

C'est ainsi que l'on peut classer les pâturages en :

\section{Pâturages de Danes et de Plateaiux.}

Ce sont les pâturages d'hivernage; aussi lour importance est-elle subordonnée au'régime des pluies. Ils comprennent :

a) Les pâturages herbacés. - Surtout utilisés par les bovidés. Sur les dunes du Nord, non loin de la vallée du fleuve, on rencontre de nombreuses graminées, de petites dimensions, plus serrées au pied des arbustes :

Le genre Pennisetum avec plusieurs espèces, donnant un fourrage ordinaire, les genres Clitoris et Aristida, assez bon fourrage, le genre Cenchrus ou Cram-Cram dont les peuplements sont très importants et constituent un fourrage passable quand la plante est jeune.

En bordure des mares, nous trouvons la même végétation herbacée, mais plus fournie et notamment le genre Andropogon à nombreuses espèces, qui donne au début de la saison des pluies un excellent fourrage. Le genre Panicum comprend aussi de nombreuses espèces dont I'une, le Panicum burgu, constitue en saison sèche, sur les bords du Niger, au bord des mares, des marigots, des terrains inondés, la seule a.imentation dı bétail.

Le Panicum Albidum, connu en Peul sous le nom de "Paguiri », est un excellent fourrage. Le Moussokoroni (Bambara) avec le Paguiri (Peul), sont les meilleurs fourrages de la région. La toxicité du Moussokoroni est assez grande au début de sa pousse. Elle disparait dès la floraison' et la fructification et devient la plante qui engraisse les moutons (Peuls). Les repousses sont aussi dangereuses.

b) Les pâturages arbustifs. - Ce sont les arbres ou arbustes (épineux) dont les feuilles et les gousses sont mangées par les moutons et les chèvres. Ils consomment d'abord les feuilles et gousses à leur portée, puis le berger coupe les branches hautes, et souvent aussi l'arbre en ontier. 
Les Mimosées sont représentées surtout par l'espèce Acacia tortilis dont les moutons sont très friands des gousses. L'Acacia vereck est surtout répandu dans le Nord où il constitue souvent la seule végétation arbustive.

Dans la région des mares, on rencontre l'Acacia seyal à fleurs en boules d'un jaune vif, très appêté des moutons, qui forme une grande partie de la végétation épineuse de la boucle du Nigcr dans sa partie Nord; l'Acacia arabica (Gonakié), feuilles et gousses très appréciées, qui vit dans le Sahara et le Nord de la zone sahelienne, et l'Acacia albida, rencontré en abondance de Tombouctou à Hombori.

Aux Légumineuses appartient aussi le genre Bauhinia dont le Bauhinia rufescens qui a des feuilles et des gousses bien appêtées du mouton et de la chèvre.

Ilśs sont utilisés à partir de juillet et leur durée est fonction de l'intensité des précipitations ațmosphériques.

\section{$2^{\circ}$ Pâturages du Fleuve ou de Décrue.}

Sans atteindre l'importance des pâturäges de décrue du delta, les rives du Niger présentent de vastes bourgouttières et champs de Baô (1).

Ces pâturages du Fleuve, bien que moins variés que les pâturages de la steppe, sont d'une grande importance, car ils conditionnent la vie des troupeaux de mars à juin-juillet.$$
*^{*} *
$$

Les déplacements des nomades du Gourma sont conditionnés par la répartition des pâturages dont il vient d'être question et la présence de l'eau. Cette recherche de l'eau et du vert donne l'explication de toutes les tribulations des éleveurs saheliens. Les déplacements s'effectuent à des intervalles réguliers et dans les directions toujours les mêmes, ce qui en fait un phénomène cyclique. D'une façon générale, il est possible de systématiser ces oscillations saisonnières en quatre périodes, réserve faite des Kel-Gossi, des Kountas et des Chioukancs.

10 Période janvier-mars. - Tous les nomades s'approchent du fleuve et s'installent sur les prernières durres. Les animaux utilisent ainsi tous les pâturages de steppes situés sur une bande de terre large de 20 à 30 kilomètres. Tous les deux jours (bovidés) et trois jours (ovidés et capridés), les animaux viennent s'abreuver au fleuve.

$2^{\circ}$ Période avril-juin. - C'est la période des pâturages dans les bourgouttières où chaque tribu possède, par droit de premier occupant, une portion de cette précieuse prairie verte. Mais comme l'utilisation n'est guère méthodique, il s'ensuit un gas-

(1) Riz sauvage. pillage tel, qu'en moins de six semaines il ne reste plus que des tiges piétinées et souillées par les déjections. Aussi assiste-t-on à un spectacle lamentable pendant les deux mois qui précèdent l'hivernage, au cours desquels les animaux errent d'une île à une autre. C'est la période où la mortalité des jeunes atteint son maximum, soit par inanition, soit par affections parasitaires ou infectieuses écloses à la suite de la sous-alimentation. Los adultes qui ne sont pas engraissés pendant l'hivernage paient également un lourd tribut.

3o Période juillet-septembre. - La situation cidessus explique le départ précipité des nomades dès que les premières pluies ont permis la pousse des herbes et la constitution des mares de relai. Bien souvent, c'est la période des grands drames résultant de l'imprévoyance des nomades qui, s'étant aventurés loin du fleuve; sans eau, se voient à la merci de la soif. Telle fut la situation des KelHormas Gourma au début de l'hivernage 1912. Cette fraction perdit plus de 500 bovidés et 1.000 moutons et chèvres, plusieurs personnes succómbèrent.

Ainsi éparpillées dans le Gourma, les diverses tribus ou fractions s'installent sur leurs points coutumiers. A partir de cette date, les déplacements diminuent en amplitude; ils s'effectuent d'une prairie à une autre suivant la nature, l'importance et la quálité des pâturages sans que les divers groupements sortent de leur zone de parcours normal.

40 Période octobre-décembre. - L'hivernage se termine généralement dès fin septembre. Vers la mi-octobre, toutes les mares de moindre importance se dessèchent. Lies nomades se retirent donc près des grandes mares : les uns, ceux qui restent toute l'année dans le Gourma, vers les mares permanentes de Gossi, Niangaye, Benzeina; Bambou, Fentroun; les autres, ceux qui descendent au fleuve, vers les grandes mares de relai d'Ikarakanten, Tiné-guenguen (tribus du centre), mares de Rachar, de Fariama, de Tintelout (tribus de l'Est), de Tiné-Tin Kart, de Mamar (tribus de l'Ouest). Puis dès que ces points d'eau commencent à tarir, les éleveurs les abandonnent pour les paturages dont il est question dans la première période.

Tel" est le phénomène pour l'ensemble des nomades du Gourma. Mais à côté de ce mouvement général, il existe un systeme de nomadisation de moindre amplitude qu'effectuent :

a) Les Kel-Gossi, une partie des Foulanes de Waghaï et Magoubé, autour de la mare permanente de Gossi

b) Les Kountas, les Foulankriabés, autour des anciens bras du fleuve (Garou, Haribongo, Kabongo, Dô, Niangaye) actuellement desséchés, mais où des puits forés par les éleveurs eux-mêmes alimentent bêtes et gens pendant la saison sèche. 
A l'exclusion des Kel-Gossi dont les bovidés utilisent le bourgou du lac Gossi, toutes les autres tribus (Kountas, Foulankriabés, Chioukanes) qui séjournent toute l'année dans le Gourma, continuent à faire paître leurs animaux dans les pâturages de steppe. Il en est de même des Kel-Antassar campés à Fontroun. C'est donc une erreur de croire que les pâturages de brousse soient peu alibiles pendant la saison sèche, les exemples pıécédents démentent formellement ce préjugé. La seule condition est l'existence des points d'eau susceptibles de permettre l'utilisation rationnelle d'abondants pâturages habituellement délaissés par manque d'eau. De ces remarques, il résulte les conclusions suivantes:

1. Bien que d'immenses pâturages restent inutilisés dans le Gourma, la majorité du cheptel souffre chaque annćc do disette;

$2^{\circ}$ Il est parfaitement possible de vivre toute I'année dans le Gourma. L'exemple des Kel-Antassar, des Kountas, des Foulankriabés confirme cette assertion;

3o Puisque le système des transhumances doit persister, il importe de réglementer l'utilisation des pâturages du Niger et d'aménager les différents relais vers l'intérieur.

Tous ces faits montrent l'opportunité de l'organisation d'un régime pastoral pour l'utilisation rationnelle des ressources fourragères, sans porter préjudice aux formations sylvestres des différentes régions.

Deux groupes de régions attirent l'attention par leur aridité quand on examine le croquis du Gourma.

a) Le $l^{\text {er }}$ groupe est constitué par une bande de terre large de 90 à 100 kilomètres séparant le fleuve Niger des grandes mares de l'intérieur. De l'est à l'ouest, cette bande, bien que riche en pâturages, est privée de points d'eau pendant huit mois de l'année.

b) Des grands espaces séparent les grandes mares de l'intérieur entre elles : par exemple : entre Bourzaye et Doro (180 km.), entre la mare de Rarouze et celle de Tintelout $(90 \mathrm{~km}$.), entre Fentroun et Gossi $(100 \mathrm{~km}$.), Niangaye et Dimamore $(175 \mathrm{~km}$.), entre Haribongo et Bembou ( $100 \mathrm{~km}$ ), entre Bembou et Niangaye $(100 \mathrm{~km}$.), etc. Toutes ces grandes étendues, bien que couvertes d'immenses réserves fourragères, demeurent inutilisées pendant sept à huit mois de l'année.

Aussi la question qui domine l'élevage, le commerce et la production industrielle du bétail est-elle celle de l'abreuvement en saison sèche. Dans toute la zone sahelienne, les déplacements saisonniers sont rigoureusement commandés par la recherche de l'eau. Il est vrai que les pasteurs préfèrent naturellement les eaux de surfacc; mais celles-ci ne persistent que dans quelques rares mares qui se trouvent, pour cette raison, très surchargées. Il en est de même des bords du Niger, témoins de contestations souvent dramatiques, et cette accumulation est la cause de la diffusion des maladies contagieuses.

Des puits disséminés en grand nombre dans l'intérieur aideraient pallier à cette situation précaire, en assurant une répartition meilleure et par suite une utilisation plus générale des pâturages.

La diminution de la transhumance qui en résulterait, conduirait insensiblement à fixer les éleveurs au sol. Ce serait peut-être un acheminement et une étape vers l'éveil du désir de la propriété foncière, levier puissant de toute richesse et de tout progrès économique et social.

$1^{\circ}$ Etat actuel du système. - Dans toute l'étendue du territoirc, il n'existe à notre connaissance que 4 puits dont 2 en mauvais état (le puits de Fentroun et celui de Bembou sont à demi comblés). Seuls fonctionnent actuellement les puits de Time-Tinkart, foré en février 1944, et de Zeigié, foré en juin 1944, sur la route d'exportation entre Benzeina et Dourgama.

En 1941, une citerne a été aménagé à Tindadénit, mais à défaut de curage elle est aujourd'hui à moitié comblée. Tel est en résumé le système actuel pour l'ensemble du Gourma.

$2^{\circ}$ Extension et zones à prospecter. - Bien que les pâturages soient abondants dans tout le Gourma, certains d'entre eux seulement doivent retenir l'attention pour le développement de l'hydraulique pastorale. Ces pâturages doivent se' trouver sur le parcours normal des éleveurs afin que leurs habitudes soient heurtées le moins possible.

Les installations ainsi aménagées devront être munies d'un systcme de poulies actionnées par des bêtes de somme, car, étant donné la profondeur des puits, il est à peine soutenable que la montée de l'eau put se faire à bras d'hornme.

La prospection devra viser deux buts :

a) Relier le fleuve avec les grandes mares de l'intérieur par un système de puits aménagés à égale distance de ces deux zones. Par exemple :

1 o Pour les tribus de l'Fist:

1 puits entre le fleuve et la mare de Bourzaye.

1 autre entre le fleuve et la mare de Tintelout en recreusant l'ancien puits de Karkassou.

20 Pour les tribus du Centre:

1 puits entre le fleuve et Fentroun.

1 puits entre le fleuve et la mare d'Ikarakarten.

1 puits entré le Sereré et Bambou.

$3^{\circ}$ Pour les tribus de l'Ouest :

1 puits entre Gcurou et la mare de Tintechak.

1 entre Raou et le lac Isșei, 


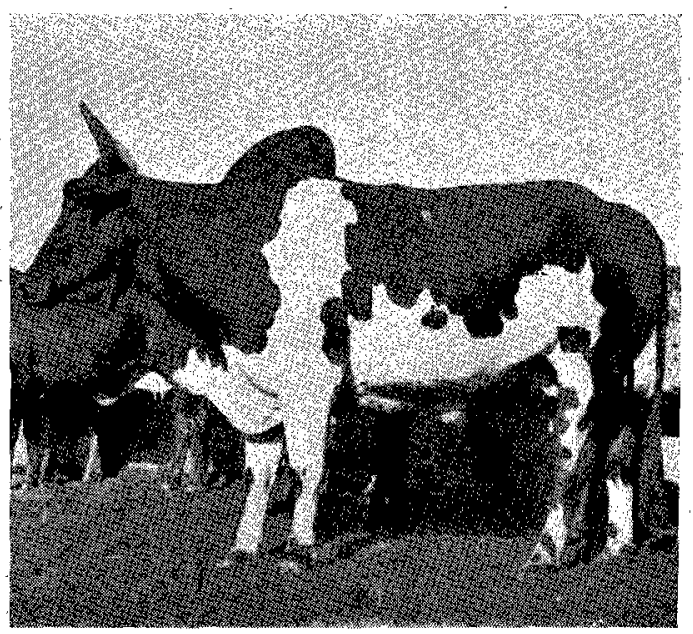

Bouf, Touareg

b) Relier les grandes mares entre elles par des puits creusés à égale distance les unes des autres. Par exemple :

10 Pour les tribus de l'Est :

1 puits entro la mare do Bourzayo ot collo de Tiguilat aux environs de la hauteur d'Alcada.

1 autre entre la mare de Rarouze et celle de Doro.

$2^{\circ}$ Pour les tribus du Centre:

I entre Fentroun et Gosisi.

1 entre Bimbou et Niangaye.

1 entre Niangaye ef Dimamou.

3o Pour les tribus de l'Ouest :

1 puits pourrait être aménagé dans la cuvette de Marmar entre Haribongo et Dimamou sur la piste commerciale.

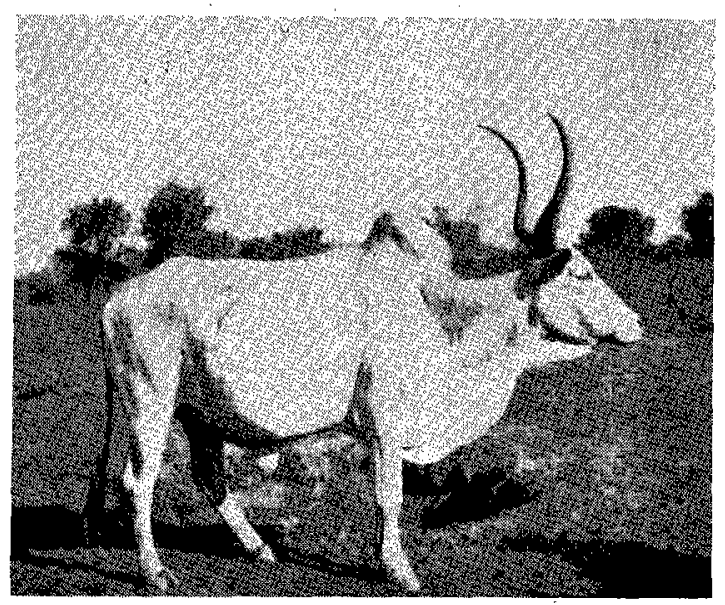

Bouf Toronké
1 autre entre Benzeina et la chaîne de montagne reliant Hombori à Douentza.

Soit au total ' 14 puits.

\section{LE CHEPTEL}

Son importance. - Te cheptel de la Boucle peut être 'évalué approximativement :

$$
\begin{array}{ccccc}
\text { Bovins Ovins et Caprins } & \text { Chevaux } & \text { Anes } & \text { Chameaux } \\
500.000 & 1.000 .000 & 10.000 & 20.000 & 1.000
\end{array}
$$

Sa répartition. - Il est réparti entre les mains d'une cinquantaine de milliers d'habitants, pour la plupart des Touareg. Celui des sédentaires est réduit.

La répartition du cheptel chez les Touareg, un peu comme chez. les Maures de Mauritanie, est fonction de leur organisation sociale qui comprend : la casto gucriòre (Imochars ou noblcs), cssontiel lement nomades, les Imrads ou vassaux, à des

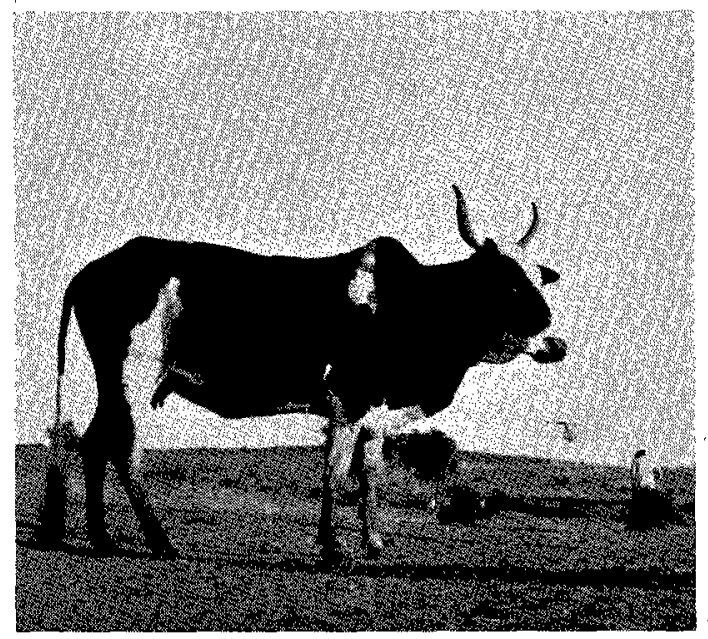

Vache Peule

degrés divers plus ou moins teints de sang étranger (maure) ou de sang noir, les tribus maraboutiques, tels que les Kel-Souk qui constituent une caste intermédiaire entre les nobles et les serfs, et les Bellahs, serviteurs noirs d'origine très disparate. Certains, fixés chez les Touareg depuis plusieurs 'générations, font souche sur place et par l'apport du sang targui constituent une race métis à type assez caractérisé, rappelant les Pourogrres de la Mauritanie. Ce sont les Bellahs qui assurent la vie matérielle des Touareg; les Bellahs soignent les troupeaux, creusent les puits, assurent le ravitaillement journalier des campements. Les uns sont attachés aux familles (Bellahs de tente), les autres vivent en tribus. Ils étaient les vrais gestionnaires des troupeaux de leurs maîtres, et en disposaient pour leurs 'besoins, à charge de fournir aux Touareg les boufs nćcossaires à l'échange contre les céréales et les 
vêtements; de plus en plus, ce rôle se limite à celui de berger, car le 'l'argui s'intéresse maintenant à ses troupeaux.

La plus grande partie du cheptel appartient soit à l'élément noble (Imochar), soit à l'élément maraboutique (Kel-Es-Souk), soit à certains Imrads. Ces divers éléments de la population ne représentent guère plus de 10 à $15 \%$ de la population.

La classe moyenne (Imrads), forgerons, Bellahs, entretient surtout des petits troupeaux de chèvres et de moutons. L'élevage de l'âne leur est généralement confié, tandis que les nobles se réservent l'élevage des bceuts et des chameaux. Le goût du cheval chez les Touareg est peu prononcé.

Le Targui est, en général, économe en ce qui concerne ses troupeaux. Il vit étroitement de son bétail qui est associé à tous les actes de sa vie, qui lui procure la viande les jours fastes, le cuir, la peau, la laine pour ses usages domestiques, le lait pour s'alimenter et engraisser sa femme. Certes, il vend

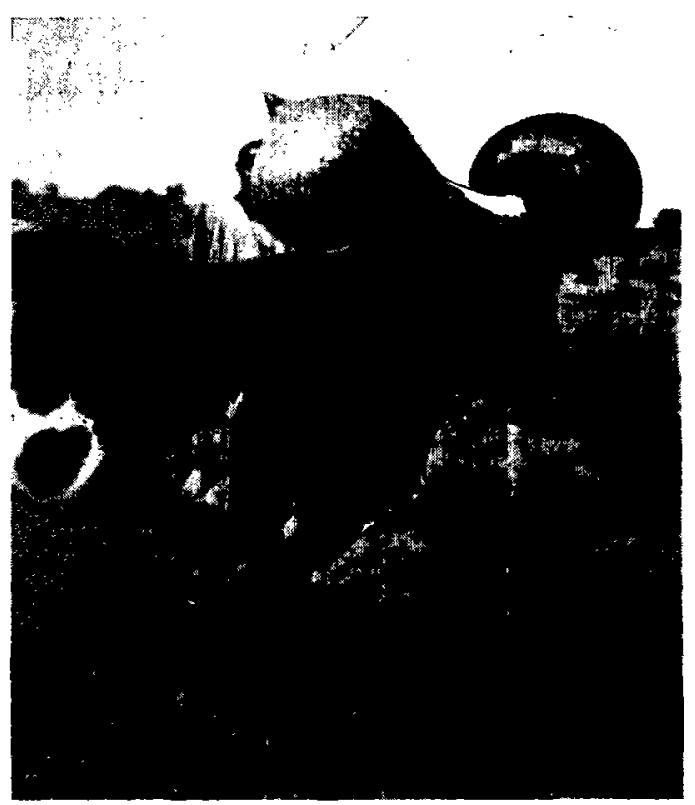

Vache du Manga

\section{a) Les bovins.}

Les bovins de la boucle sont des zébus de la race Targui.

Le zébu Targui est un zébu à cornes courtes, d'une taille moyenne de $1 \mathrm{~m} .25$ chez le taureau, $1 \mathrm{~m}$. 20 chez la vache, $1 \mathrm{~m}$. $35 \mathrm{chez}$ le bouf, avec un poids correspondant de 300 à 325 kilos, 275 à 300 kilos et 350 à 450 kilos. La robe est très variée car les troupeaux ne sont pas homogenes. Elle est le plus souvent dans la gamme des foncés : noire, pie noire, pie rouge. Les robes aubères et blanches se rencontrent.

La tête est à profil rectiligne avec des cornes courtes (28 à $30 \mathrm{~cm}$.). un corps bien charpenté chez les bons sujets, une poitrine spacieuse, près de terre, un dos large, un rein soutenu, une croupe inclinée et forte, une culotte bien dessinée, des membres solides. Comparé aux zébus peuls ou maures, il est moins enlevé, plus épais.

Aptitudes. - Bon animal de boucherie qui donne sur place et en état un rendement de $48 \%$.

Bon animal porteur, mais beaucoup moins utilisé que le zébu maure.

Aptitude laitière peu marquée. Mamelles et pis peu développés. 


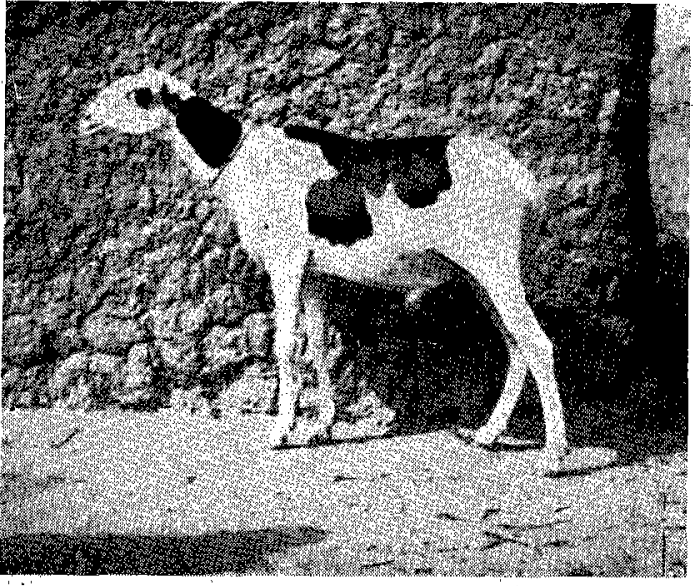

Mouton Maure

Le troupeau. - Le troupeau bovin est presque en entier entre les mains des tribus Touareg. Les Songhaîs, malgré le peu de soins qu'ils apportent à leur bétail, augmentent cependant leurs troupeaux par suite de l'échange des animaux des nomades contre leurs grains. Parmi les tribus à cheptel bovin important, citons dans la Subdivision de Rharous, les Irréguenaten avec plus de 100.000 têtes, les Chioukanes de l'Ouest et les Foulankriabés avec environ 50,000 têtes; les Kel Cossi avec 25.000, les Iguadaren I avec 20.000, les Imededagan du Seréré avec 15.000 .

La composition moyenne des troupeaux est la suivante :

Taureaux et boufs........ 15

Vaches ............... 55

Jeunes et veaux........... 30

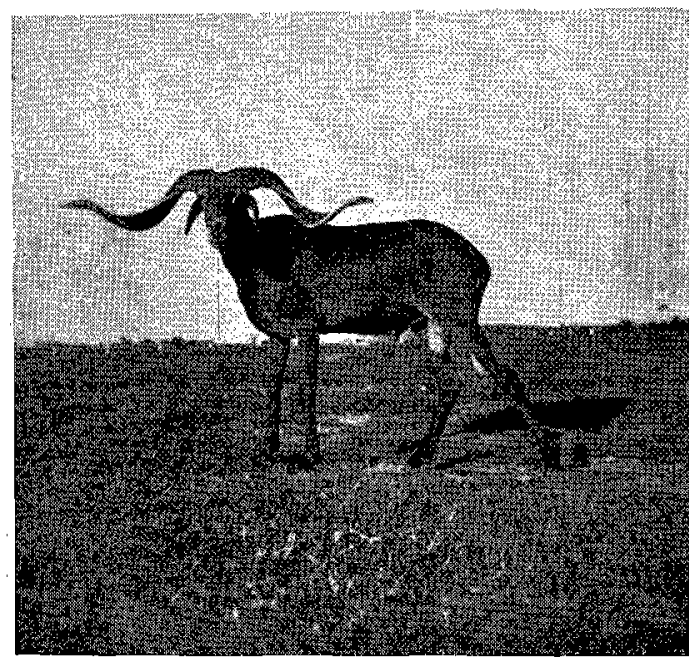

Bélier Sambourou

\section{b) Les moutons et les chèvres.}

Le recensement officiel, c'est-à-dire celui des animaux déclarés pour l'impôt, est pour la seule Subdivision de Rharous de 650.000 têtes. On peut l'estimer au moin's à 800.000 et accorder au minimum 200.000 lêtes pour la partie du cercle de Gao située sur la rive du Gourma.

Dans l'ensemble; le troupeau ovin est moins important que le caprin et n'atteint que les $2 / 5$ du total. Le petit bétail rencontre dans cette région des conditions climatiquies qui lui sont très favorables; il est moins exigeant que le gros bétail au point de vue des pâturagẹs, pas plus au point de vue abreuvement.

Moutons et chèvres sont représentés :

les premiers par les moutons à poil et les moutons à laine;

les seconds par les chèvres du Sahel.

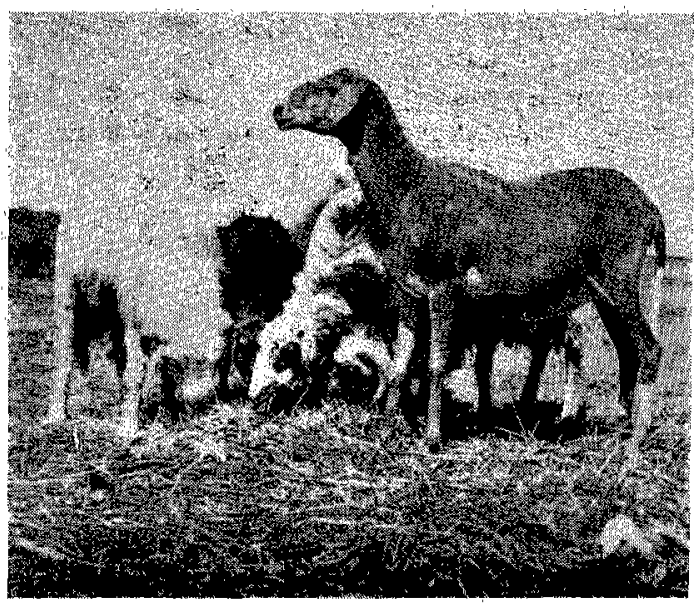

Brebis Touareg et moutons du Macina

\section{Les moutons.}

Moutons à poil. - Le type dominant est le petit mouton Targhi dit du. Gourma, par opposition du. grand mouton Targui du Haoussa (Araouane-Kidal). Il est d'une taille variant entre $0 \mathrm{~m}$. 65 et $0 \mathrm{~m}$. 75, sous poils feutrés à l'avant-main, le plus souvent roux, mélangés plus ou moins de blanc. Son poids moyen est de 30 à 40 kilos.

Le Targui est un mouton du Sahel, c'est-à-dire un animal peu exigeant, sobre, apte à la transhumance. Il se différencie du mouton Maure ou Toronké par sa silhouette moins enlevée, moins longiligne. Il est mieux conformé pour la boucherie. Le bélier a un front plat, un chanfrein très convexe, des cornes en spires lâches se terminant par un crochet vers l'extérieur. Les pendeloques sont presque constantes, le garrot est saillant, le dos droit, court, la croupe large et oblique, la queue atteignant à peine 


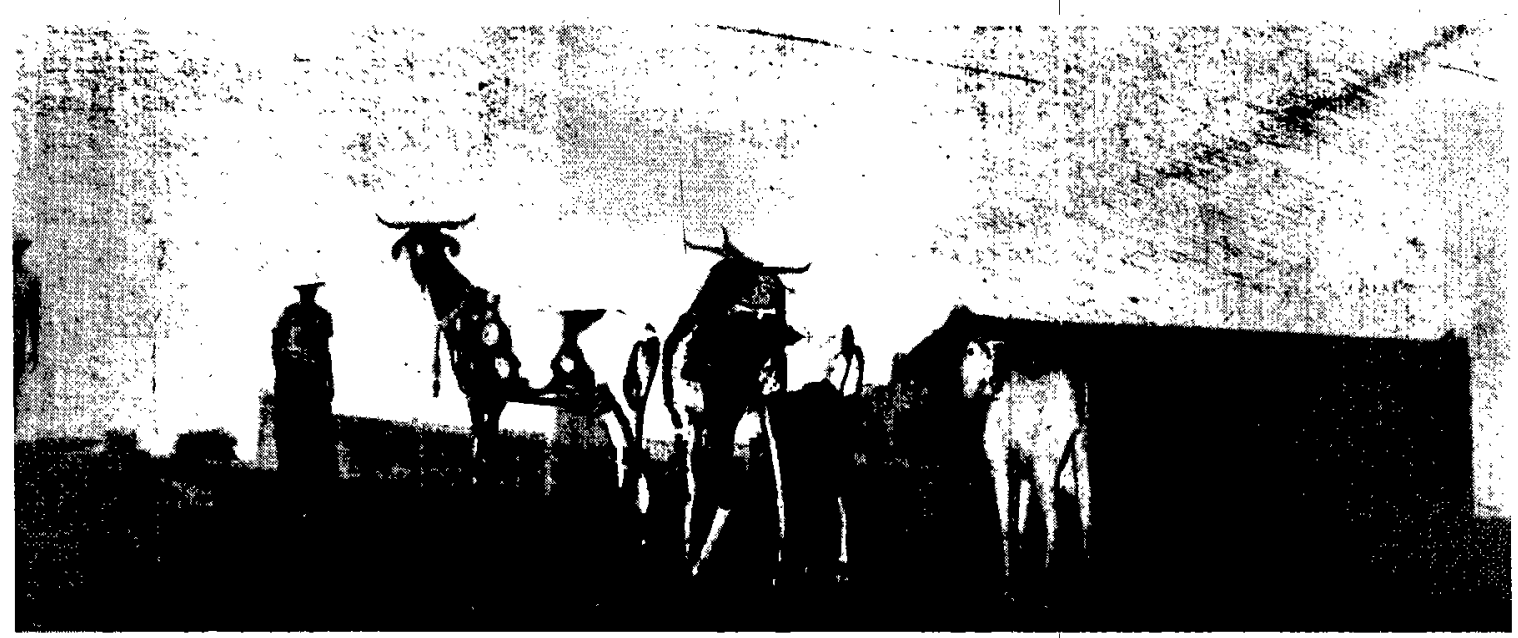

Chèrres de la boucle du Niger

la pointe des jarrets. Les membres sont forts, les sabots larges comme tous les saheliens. Il possède comme les Maures et les Djallonkés un fort camail allant de la gorge à l'interars.

La brebis est fine, moins grande, moins lourde, avec des rudiments de cornes et une mamelle -assez développée.

Le mouton est élancé, fin, bien conformé pour la boucherie; il atteint une taille de $0 \mathrm{~m} .75$ à $0 \mathrm{~m} .80$ et un poids de 45 à 50 kilos.

La castration des béliers est régulièrement pratiquée et le Targhi ne conserve que les reproducteurs indispensables à son élevage. La lutte est réglée dans certaines tribus, de façon que l'agnelage ait lieu en bonne saison.

Aptitudes. - Le mouton Targhi est un bon animal de boucherie dont le rendement est de 45 à $47 \%$. Il est aussi utilisé dans les tribus pauvres au transport de l'eau.

La brebis bonne laitière est traite deux fois par jour, matin et soir. Le lait sert à l'alimentation des nomades et en hivernage, la surproduction à la préparation des fromages.

\section{Les chèvres.}

La tête est petite à front plat, à chanfrein rectiligne ou subconcave, à face courte et barbillon. Les cornes sont assez longues chez le bouc, épaisses, spiralées, dirigées en arrière et divergentes. Celles de la femelle sont fines, courbées en arrière. Le cou est mince et long, le garrot et le dos saillants, la poitrine étroite, la croupe courte, inclinée, la queue courte et relevée, les membres sont longs avec des sabots larges.

La robe est très mélangée, bariolée, souvent tricolore. Les poils sont ras. Le bouc porte une crinière s'etendant parfois jusqu'à la croupe.

Aptitude. - Viande : celle des moutons du Sahel.

Lait : Les chèvres ont des mamelles bien développées, bien descendues, avec deux pis en bouteille. Ce sont de bonnes laitieres qui sont traites.

Le troupeau ovin et caprin. - Ce troupeau est aux mains des nomades, surtout de la classe moyenne, qui préfère la chèvre à cause de son lait plus abondant.

Parmi les tribus de Rharous possédant un fort cheptel, signalons le Irregueneten avec' 400.000 têtes, les Kel-Gossi avec 80.000 , les Chioukanes et les Foulankriabés 75.000 , les Iguadaren 150.000 , les Imededagane de Sereré 50.000, les Kóuntas 20.000, les Iguadaren II, 15.000, les Kel Temoulait 15.000.

\section{Les chevaux.}

Les Touareg ne sont pas, comme les Maures, des éleveurs ni des connaisseurs de chevaux. Ceuxci sont élevés par les sédentaires du fleuve, à la façon du bétail, c'est-à-dire à l'abandon dans les bourgouttières. C'est le cheval Songhaï, dont les caracteres ethniques chez certains sujets rappellent ceux des poneys autochtones de l'A.O.F., mais son habitat au confluent des deux zones d'influence de nos deux grandes races principales, barbe et dongolaw, et son mode d'élevage en ont modifié le type.

En parlant d'un petit cheval, les indigènes de Douentza Hombori disent « il est court comme un cheval Targui ». Assez commun dans son ensemble, souvent décousu, il se présente généralement avec une tête longue à profil rectiligne, une encolure grêle, un dos long, une poitrine étriquée, une croupe tranchante, des membres faibles, des sabots larges. Cependant nous avons rencontré de beaux 


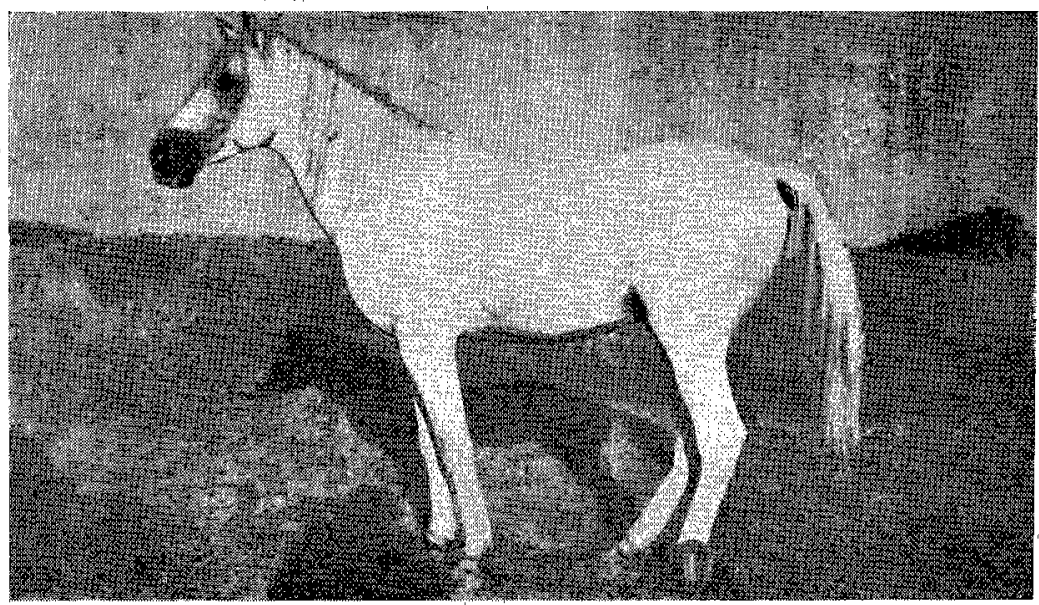

Cheval type Arabe-barbe

et ventre gris sont peu nombreux et rappellent les ânes marocains. Les ânes blanc creme avec ou sans raie cruciale sont assez rares.

Le troupeau. - Environ 12.000 têtes dans la Subdivision de Rharous, dont la presque totalité chez les Touareg. Parmi les plus riches, citons toujours la tribu des Irreguenaten (5.000), de Chioukanes de l'Ouest et des Foulankriabés (1.700), des Iguadarem I (2.000); des Kel Gossi (1.000), des Kountas (600).

Parmi les sédentaires, seuls ceux des cantons: de Sereré

modèles, bien proportionnés, se rapprochant du M'Bayar.

La taille varie de $1 \mathrm{~m}$. 25 à $1 \mathrm{~m}$. 35. La robe est presque uniformóment bai ordinaire avec ventre lavé et sans particularité.

Les chevaux importés du Sahel ou du Niger se recomaissent facilemenl.

Le troupeau. - Le troupeau chevalin du'fleuve est assez important pour mériter à la région le nom de zone d'élevage. Il atteint environ' 10.000 têtes réparties sur les rives et dans les îles du fleuve, de Tombouctou à Ansongo. Parmi les éleveurs sédentaires, citons dans la Subdivision de Rharous les cantons de Sereré (250), de Rhergo (350), dans 'la Subdivision de Bourèm, le canton de Bourèm (1.000), de Bamba (300), de Takambo (250), au total dans cette Subdivision 3.000 , animaux dont 1.700 juments, 700 chevaux, 600 poulains. La Subdivision de Gao en possède environ 1.500 et celle d'Ansongo 500.

Parmi les nomades, citons les Irreguenaten (250), les Chioukanes de l'Ouest et les Foulankriabés (350), les Iguadaren I (100): En général, lorsque les Touareg quittent les pâturages de décrue, ils confient leurs chevaux aux sédentaires.

\section{Ines.}

L'âne, animal de bât, est presque indispensable au nomade du Sahel, aussi est-il très apprécié par lui. Les Touareg emploient moins le bœuf porteur que les Maures, et chaque tente entretient des ânes.

L'âne du Gourma est l'âne du Sahel, de petite taille, 1 mètre à $1 \mathrm{~m}$. 10, il a une tête longue, lourde, chargée avec un front large et bombé, un dos long, une croupe courte, des membres secs et robustes.

Son poil est court, à robe gris cendré le. plus souvent avec raie cruciale fóncée et zébrures fréquentes alux membres. Les ânes à manteau brun et de Bambara-Maoundé possèdent un troupeau dépassant la centaine.

Sur la rive droite de la branche descendante du fleuve, dans le Cercle de Gao, le cheptel asin atteint 800 têtes.

\section{Les chameaux.}

Les tribus du Gourma possèdent un cheptel camelin peu important, à peine un millier de têtes, car cet animal vit mal au-dessous du fleuve. Seuls, ceux qui sont acclimatés peuvent tenir, et un chameau de l'Azaouad transplanté brusquement dans le Gourma est destiné à périr rapidement. La maladie de la mouche (M'Bori), diverses infestations dues à l'abreuvement aux mares souillées; des pâturages différents en sont' les causes principales.

Le troupeau est aux mains des nomades. Parmi ceux de Rharous; citons les Irregueriaten (250), les Kountas (70); les Iguadaren I (60).

\section{EXPORTATION DES PRODUITS DE L'ÉLEVAGE}

\section{a) Disponibilités.}

1 o Bovidés. - L'expérience des exportations de bétail acquise par le Service de l'Elevage ainsi que la composition moyenne des troupeaux nous donnent le taux approximatif de nos disponibilités en bovins de boucherie (bouf au-dessus de 5 ans et vaches hors d'âge). Il est environ le 1/20 du troupeau, soit 25.000 têtes.

Ce taux faible est dû :

a) au mode d'élevage; le nomade ne vend son bétail qu'en fonction de ses besoins; ils sont réduits et il ne peut que difficilement se procurer les denrées qui lui sont nécessaires : thé, sucre, étoffes;

b) au peu de précocité de nos animaux; le bœuf n'atteint son plein développement qu'à 6 et 7 ans: 
c) à la mortalité due aux affections enzootiques et épizootiques;

d) aux troupeaux incessibles.

Moutons et chèvres. - Le nomade n'apporte pas à son petit bétail le même intérêt qu'au gros, il le vend plus facilement, il l'exploite plus rationnellement et la capacité de renouvellement du troupeau өst beaucoup plus grande. Le mouton est apte à la boucherie à ? et 3 ans, et la natalité dans les troupeaux atteint $75 \%$ des femelles alors qu'elle n'est que de 50 à $60 \%$ chez les bovins. Le milieu se prête mieux au petit élevage qui trouve dans les pâturages arbustifs une alimentation suffisante. Enfin les épizooties propres aux deux espèces sont rares et la principale cause de mortalité est due aux parasitoses contractées dans les bourgouttières. On peut évaluer les disponibilités au l/4 de l'effectif, soit 250.000 têtes.

\section{b) Commerce de bétail.}

Le commerce local est très restreint. C'est ainsi qu'en 1944, à Rharous, le centre le plus importany. on a abattu 296 bovins, 50 ovins, 36 caprins. Les saisies effectuées montrent bien le degré d'infestation parasitaire de ce bétail : elles ont porté sur 232 foies à distomatose et 21 cas de cysticercose localisee.

Marchés au bétail. - Il n'y a pas de véritables marchés au bétail dans le Gourma, mais à sa périphérie. Chaque chef de tente conduit à son marché préféré, situé le plus souvent dans les régions avoisinantes et d'après les renseignements qu'il possède sur les cours pratiqués pour les bœufs. moutons, chèvres nécessaires au paiement de l'impôt. Par fierté, il marchande peu et vend son bétail moins cher que le Peul et le Maure.

Ce n'est qu'à partir de ces marchés initiaux que les routes d'exportation prennent naissance. Hombori, par sa situation privilégiée, est le marché d'exportation le plus important de la Boucle durant l'hivernage. N'Gouma et Konna (Cercle de Mopti) sont surtout fréquentés par les tribus de l'Ouest et Gao par celle de l'Est.

Exportations. - De ces centres partent les différentes routes d'exportation :

a) Vers le Niger et la Nigeria anglaise par la route du fleuve;

b) Vers la Gold-Coast et la Côte d'Ivoire par Hombori Djibo, Kaya, Pouytenga, Tenkodogo, Bittou, Bawku.

Les exportations étant souvent clandestines, l'importance de ces mouvements nous échappe, mais ils doivent être, certaines années, voisins de nos disponibilités.

\section{c) Cuirs et peaux.}

Nous avons vu que la consommation locale est réduite. Elle porte principalement sur les abatages familiaux de chères et de mouton's. L'industrie locale des peaux est active dans le Gourma et absorbe de nombreuses dépouilles. Ceci découle des besoins de la vie nomade; matériel de campement (tentes! cordes, delous, guerbas) et du goût prononcé de ceux-ci pour les objets ne cuirs : coussins, fourreaux de sabre, gris-gris; etc.

Aussi la production de cuirs et peaux de la région est peu importante; elle atteint à peine le $1 / 10$ de la production du Soudan : 4 à 5 tonnes.

\section{d) Laine.}

La production lainière de cette région est faible et en grande partie utilisée par l'artisanat local pour la confection de couvertures.

Lors, des foires à laine tenues à Rharous ces dernières années, le tonnage présenté a été de l'ordre de 3 à 4 tonnes dont $50 \%$ en standard $n^{\circ} 1$, $40 \%$ en standard $n^{\circ} 2$ et $10 \%$ en standard $n^{\circ} 3$.

\section{e) Lait.}

Étant donnée l'importance des troupeaux, la production de lait est élevée malgré le peu de qualité laitière des femelles, mais l'exploitation de ce produit ne peut être assurée que lorsque le bétail est rassemblé dans les bourgouttières du fleuve. Il en est de même pour la production du beurre. Celle du beurre fondu pratiquée par les indigènes est importante et s'écoule sur les marchés d'Aghalal, Modyakoi, Bambara-Maoundé. Rharous, Hombori. On peut l'évaluer à 25 tonnes.

La fabrication du fromage targui a lieu durant l'hivernage et donne lieu à un petit commerce local.

\section{AMÉLIORATION DE L'ÉLEVAGE}

Nous pouvons définir le Gourma, une région riche en pâturages, mais dépourvue pratiquement d'eau' durant six mois de l'année, de janvier à juillet. Il paraît donc inutile d'entreprendre l'amélioration du bétail sans en améliorer les conditions de vie, répéter l'erreur que nous avons souvent commise.

Notre cheptel est parfaitement adapté à ce milieu sahelien; il constitue avec les nomades une véritable symbiose que nous ne devons pas rompre. Vouloir lui demander à l'heure actuelle plus de viande, de lait, de laine est une utopie. Nous devons nous contenter d'en augmenter le nombre en diminuant la morbidité et la mortalité de nos troupeaux. Plus tard, mais bien plus tard, nous améliorerons les individus.

Cette mortalité des troupeaux est due en ordre principal aux affections épizootiques, telles la peste bovine, la péripneumonie, en ordre secondaire à la sous-alimentation qui, aux époques de disette, 
réveille toutes les affections latentes, toutes les parasitoses dont l'animal est porteur.

Quant aux mauvaises conditions d'hygiène et d'entretien, telles le prélèvement de lait au détriment des jeunes, le manque de sélection, d'abris, l'abreuvement aux mares souillées, les pertes par les fauves, lé peu de soin apporté aux produits animaux, elles sont la conséquence du mode de vie, de la mentalité des nomades et ce ne sont pas les palabres qui y porteront remède:

La lutte contre les épizooties est possible; nous possédons maintenant sérums et vaccins pour les combattre, mais encore faut-il que le pays soit équipé. Nous, sommes en pays nomade et le seul moyen de locomotion est le chameau, le seul point d'arrêt est le puits ou la mare. Transporter du matériel pour la préparation du vaccin antipestique dans ces conditions est une gageure, réaliser une vaccination correcte est un tour de force. Aussi notre action sanitaire dans cette région est à peine amorcée, malgré la demande des éleveurs. La construction d'un centre vétérinaire important à Rharous avec fabrication des vaccins et sérums sur place et utilisation à la période favorable, c'est-àdire à l'époque où la plupart des troupeaux sont rassembles le long du fleuve, permettra de remédier à cette situation.

Quant à la sous-alimentation qui est à l'origine des nombreuses déficiences et mortalités, nous en avons étudié les causes et les moyens de la combattre. Ils paraissent simples à première vue : une meilleure et plus longue utilisation des pâturages grâce à des points d'eau permanents; mais sur place on juge différemment. Les résultats obotenus ces dernières années ne sont guère encourageants; des puits profonds en terrains durs sont sans eau, des mares aménagées sont comblées. Il y a.certainement de notre part un manque de compétence, de moyens. Une telle entreprise qui devrait apporter la richesse dans le pays ne peut être confiée qu'à des spécialistes. Une étude géologique préliminaire du soussol est indispensable avant d'aborder la construction des points d'eau et celle-ci doit être conduite avec des moyen's matériels qui nous manquent. Les ressources en eau du sous-sol du Gourma, si l'on en juge par les quelques puits existants, ne paraissent pas suffisantes pour assurer dans de bonnes conditions l'abreuvemen du bétail. C'est pourquoi nous avons établi le projet d'une station de pompage, installée sur le bord du fleuve, au sommet de la' boucle, refoulant l'eau du fleuve dans un pipe-line central, desservant des pipeslines secondaires sur deux ou trois lignes parallèles au fleuve et alimentant un abreuvoir tous les 40 kilomètres.

Ce système d'adduction d'eau n'offre pas les inconvénients de l'exhausse de l'eau des' puits par des moyens mécaniques (éoliennes, pompes diverses, avec ou sans moteur); supprime tous les inconvénients des mares aménagées et des citernes: toutes les parties délịcates, en quelque sorte l'âme' du système, sont centralisées en un seul point où il sera facile de fixer un personnel technique chargé de la marche et de "l'entretien.

Telles sont' les possibilités d'équipement hydraulique que possède le plateau du Gourma. Mais à l'est et à l'ouest de ce plateau, le problème de l'eau paraît moins difficile à résoudre. A l'ouest, c'est la région des grandes mares alimentées irrégulièrement par le fleuve et que quelques aménagements simples pourraient rendre permanentes. A l'est, au sud de Gossi, de Doro, les dallols de la rive droite pourraient en certains endroits être barrés pour élever le plan d'eau et prolonger de quelques mois l'abréuvement du bétail.

D'autres facteurs, mais secondaires, peuvent encore concourir à l'amélioration du cheptel; ce sont la lutte contre les fauves, facile avec les appâts empoisonnés, l'organisation de routes caravanières, des foires, el des marchés, la sélection, Quant à l'amélioration de l'individu lui-même par l'introduction de races perfectionnées, elle n'est pas encore possible. Lés quelques essais que nous avons faits chez les Songhaïs, pour améliorer les troupeaux lainiers par la cession de béliers $1 / 2$ et $3 / 4$ sang Mérinos, ont tous échoué. 